\title{
Respuestas desde el Derecho del Trabajo a la crisis económica mundial ${ }^{(*)}$
}

\section{Luis Mendoza Legoas}

Abogado por la Pontificia Universidad Católica del Perú.

Especialización en Derecho del Trabajo por la Universidad de Castilla-La Mancha. Miembro del Grupo de Bologna.

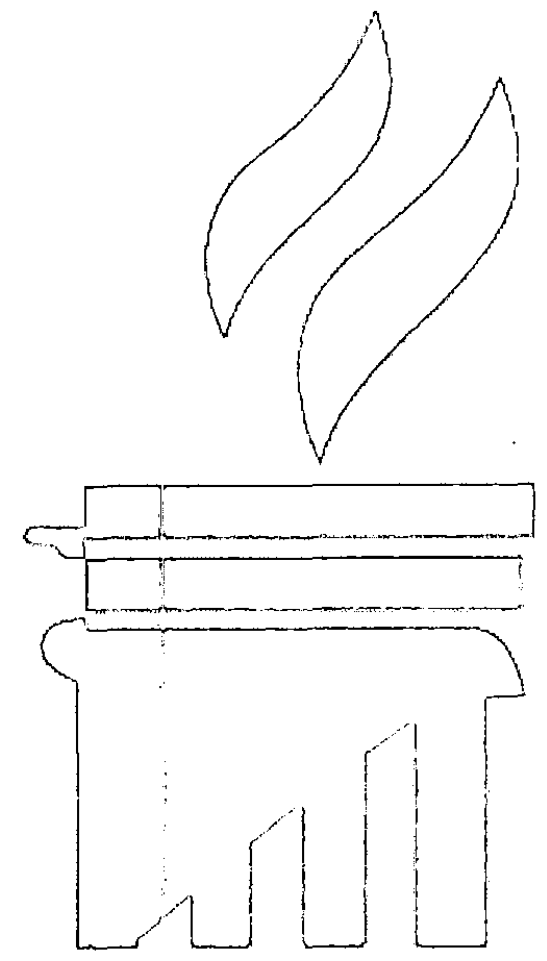

SUMARIO:

I. Erosiones en el derecho del trabajo de hoy:

1. La descentralización productiva;

2. Sofisticación técnica, capacitación y empleabilidad;

3. Proliferación de contratos temporales.

II. Notas sobre la globalización de la crisis económica contemporánea.

III. Viejas y nuevas respuestas desde el derecho de trabajo a la crisis económica:

1. Fomento del diálogo social;

2. Reducción del tiempo de trabajo;

3. Incentivos para no despedir;

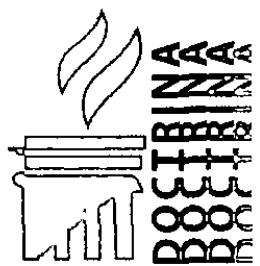

4. Suspensión perfecta de contratos de trabajo como alternativa al cese del vínculo;

5. Impulso a la constitución de microempresas y de trabajos autónomos;

6. La compensación por tiempo de servicios;

7. El caso de los programas de empleo temporal.

IV. Perspectivas para el futuro. 


\section{EROSIONES ${ }^{1}$ EN EL DERECHO DEL TRABAJO DE HOY}

La interacción del Derecho del Trabajo con los períodos de crisis económica no es nueva en la historia. Sí lo es, en cambio, el contexto globalizado en el que se produce la actual crisis económica. Esto importa una interesante variable al panorama de las relaciones laborales en el mundo globalizado de hoy. ¿Qué alternativas pueden encontrarse dentro del contexto de crisis económica? ¿Deben moderarse-al menos por un tiempo- las pretensiones de crear trabajo decente en el mundo? Sin lugar a dudas nuevos desafíos al Derecho del Trabajo se presentan en el panorama mundial.

Con la crisis ya en nuestras puertas, y sin saber en qué medida nos veremos afectados por ella, puede ser útil revisar cómo ella actúa en algunos de los rasgos característicos del erosionado Derecho del Trabajo.

\section{La descentralización productiva}

La evolución en la forma de producir presenta un panorama complejo. Las empresas de antaño tenían una estructura cerrada en sí misma, construida sobre un sistema de producción lineal (conocido como sistema de producción fordistataylorista). En cambio, las empresas hoy en dia están interconectadas por vínculos múltiples y complejos, teniendo la oportunidad de afrontar con mejores herramientas un contexto recesivo: su organización flexible y una adecuada gestión le permiten ajustar su propia producción ante la caída de la demanda.

Como ha señalado Sanguinetti ${ }^{2}$, mediante la descentralización la empresa principal busca aprovechar la mayor capacidad de respuesta de las pequeñas y medianas empresas al cam- bio económico, sirviéndose de su actividad como eficaz complemento de la que se ha decidido ejecutar en forma directa. Por eso, en un contexto económico adverso, las empresas contemporáneas están, de alguna forma, mejor preparadas para enfrentar este tipo de contingencias, desde el punto de vista organizativo.

Sin embargo, un punto negativo para las empresas de hoy es que aquella interconexión que se presentaba como beneficiosa es, al mismo tiempo, un mal si se toma en cuenta que en múltiples casos, con la interdependencia económica estructural, los efectos de la crisis podrían propagarse rápidamente en toda la red de empresas. Por efectos de la crisis la vinculación económica de las empresas globalizadas es virtud al mismo tiempo que un defecto.

Los trabajadores antes "expulsados" de las empresas principales tienen formalmente los mismos derechos que aquellos que se mantienen dentro, sin embargo en la realidad se observa cómo sus niveles de protección disminuyen en proporción directa a la menor dimensión de las empresas satélites que los contratan. Los riesgos aquí son claros: las empresas descentralizadas de modestas dimensiones pueden ser las primeras en sucumbir a los efectos de la crisis, pudiendo cerrar sus puertas en cualquier momento.

Por ello, fórmulas como la responsabilidad solidaria podrían ver revitalizada su importancia en el contexto de la crisis: los trabajadores iniciarán procesos de recuperación de los créditos laborales adeudados mediante este mecanismo, empujados por la ausencia de créditos de financiamiento para actividades propias como vivienda, educación, nuevos emprendimientos, entre otros. Con todo esto la empresa principal tendrá que asumir los costos de los que antes

1 Utilizamos este término que nos parece bastante descriptivo respecto a los procesos que han configurado al Derecho del Trabajo de nuestros días: la globalización económica e ideológica, la flexibilización y la individualización de las relaciones laborales.

2 SANGUINETTI, Wilfredo, La empresa red y el Derecho del Trabajo. En: Revista Derecho del Trabajo, No. 4, Fundación Universitas, Barquisimeto, 2007, p. 90. 
se había desprendido, presentándose serias contingencias, digámoslo así, colaterales de la crisis. Y el círculo vicioso que no acaba aquí.

\section{Sofisticación técnica, capacitación y empleabilidad}

La crisis económica tiene un importante influjo aquí también. Quienes hayan obtenido en el pasado capacitaciones financiadas por su empleador o por su propia cuenta tendrán sin duda mejores perspectivas de conservar el empleo, aunque no llegue esto a ser esto una auténtica garantía de estabilidad en el trabajo.

Por otro lado, con los previsibles ajustes en los presupuestos de las empresas, la capacitación técnica del personal tendrá un estancamiento. Los trabajadores jóvenes serán los más afectados, ya que ellos habrán tenido menores oportunidades de desarrollar estudios técnicos complementarios a su formación. Esto resulta gravísimo, pues desde ya los jóvenes sufren en mayor medida el desempleo, con lo cual, incluso superada la crisis, esta generación tendría aún un mayor número de jóvenes desempleados.

\section{Proliferación de contratos temporales}

Esto, que en la experiencia regional es bien conocido, puede tener una interpretación ambivalente en los tiempos de recesión económica. Así, si por un lado pone en manos del empleador más libertades para contratar a trabajadores temporales, perjudicando así a la estabilidad en el empleo, también es cierto que la demanda de trabajo puede ser atendida en forma más amplia, cualitativamente, si se toma en cuenta que tales los contratos temporales permiten cierta "rotación" de los puestos de trabajo, existiendo por ello mayor número de ocupados dentro de un período de tiempo.
Esta forma de contratación resulta muy importante en contextos económicos adversos, pues comúnmente los gobiernos toman medidas contra la crisis en este sentido. Estudiaremos esto con mayor desarrollo cuando analicemos a los programas temporales de empleo.

\section{NOTAS SOBRE LA GLOBALIZACIÓN DE LA CRISIS ECONÓMICA CONTEMPORÁNEA}

Desde los últimos meses de 2008 las agencias de noticias han reportado las secuelas de la crisis en todo el mundo. ${ }^{3}$ Las empresas más grandes han visto sus cimientos conmovidos ante la caída de la demanda a nivel global. El grupo finlandés Nokia, el mayor fabricante de teléfonos móviles, realizó a fines de 2008 un total de 615 despidos, aunque están confirmados más despidos para el año 2009.

Medidas más drásticas ya han sido anunciadas por otras empresas como Sprint Nextel, que en el 2009 eliminará un total de 8 mil empleos. Noticias similares se reciben de las firmas Philips, Sony y Samsung electronics. La fabricante de aviones Boeing anunció que se desharía del $6 \%$ de su plantilla. En cifras-hombre, esto significa que 10 mil de sus trabajadores perderán su empelo.

En diciembre de 200 la francesa Renault suspendió por once días a mil empleados en Argentina, cerca del $70 \%$ del total de sus trabajadores en ese país. Caterpillar despidió ya a 20 mil trabajadores, mientras que Microsoft, el gigante de la informática, ha previsto suprimir 5 mil puestos de trabajo en el 2009.

Las estadísticas nacionales no brindan una visión más alentadora. En Japón el Ministerio de Trabajo ha estimado que, antes de marzo de 2009, más de 400 mil trabajadores temporales del sector de la manufactería habrán perdido

3 Las referencias que siguen son extraídas de noticias periodísticas recibidas de información difundida entre los miembros del Grupo de Bologna, y en otros casos han sido noticias publicadas en el diario El Comercio (Perú) entre setiembre de 2008 y enero de 2009. Ver por todos: www.elcomercio.com.pe. Es importante señalar que mientras se escribía este trabajo esta sección era actualizada permanentemente con nuevas noticias sobre despidos en el mundo. Las cifras también eran actualizadas, creciendo en algunos casos de cientos a miles de despidos. 
sus puestos de trabajo. Mientras tanto, nada parece detener al crecimiento del desempleo en España: los por ellos, desocupando así a los inmigrantes que engrosan las filas de los sin empleo.

En los Estados Unidos de Norteamérica, los economistas han previsto que en 2009 la tasa de desempleo supere el $11 \%$ (en 2006 esta había sido de $4.6 \%$ ). En cifras reales, en noviembre de 2008 se dieron 181 mil despidos en todo el país, alcanzando así su nivel máximo en casi siete años.

Solo en el mes de diciembre de 2008 más de 600 mil personas fueron despedidas en Brasil. Como si esto fuera poco, un tercio del total de empresas brasileñas evalúa la posibilidad de realizar más despidos durante el 2009. De acuerdo a las estadísticas de la Dirección del Trabajo de Chile, solo en el primer mes de 2009 unas 150 mil personas perdieron su empleo.

En el Perú, el crecimiento económico precedente ha llevado a los más optimistas a señalar que existen posibilidades de enfrentar la crisis económica con relativo éxito. Sin embargo, ya es posible advertir consecuencias consumadas de la crisis en el país: las compañías mineras no están renovando los contratos temporales de los trabajadores, con lo que entre noviembre de 2008 y febrero de 2009 los desempleados del sector minero sumaron casi siete mil.

En vista de lo reseñado, pueden advertirse efectos que se están manifestando entre nosotros. Para los países latinoamericanos la crisis se traducirá, en primer lugar, como la desaceleración del crecimiento del empleo que se venía experimentando desde hace unos pocos años. Entre las causas de esta desaceleración -que podría convertirse en una estrepitosa baja si los estados no aplican políticas acertadas- está el inicio de un período de retroceso del comercio internacional y suspenso de los proyectos de inversión de las grandes empresas. Desde ya, se ha sabido de varios proyectos contemplados en los presupuestos de las empresas para el 2009 que han entrado en suspenso.
Todo esto conlleva a la reducción de las exportaciones, las mismas a las que nuestros gobiernos dirigieron sus esfuerzos en los últimos años, en procura de acuerdos comerciales de apertura a nuevos mercados. Y todo esto, ante la resignación de miles de trabajadores que deben abandonar sus puestos de trabajo, pues de la noche a la mañana son considerados "caros" o simplemente "riesgosos" dentro del planeamiento salarial de las empresas.

Con este vistazo podemos determinar cómo se configuran algunas coordenadas de los actuales y los probables impactos de la crisis económica en el mundo del trabajo.

\section{VIEJAS Y NUEVAS RESPUESTAS DESDE EL DERECHO DEL TRABAJO A LA CRISIS ECONÓMICA}

Como se ha visto, la crisis tiene un poderoso efecto sobre las relaciones laborales: existen múltiples efectos perniciosos tanto en el acceso al empleo como en la vigencia de la relación de trabajo y, desde luego, en la extinción del vínculo laboral. En lo que toca a la dimensión jurídica, la crisis ya está ocasionando reducciones en las nuevas contrataciones y en las renovaciones de los contratos temporales. Al mismo tiempo, los despidos justificados en la crisis económica están a la orden del día.

Asimismo, los beneficios adicionales (bonos por productividad o incrementos de salario) son restringidos hasta casi su suspensión. Esto es afirmado categóricamente por la Organización Internacional del Trabajo (OIT), que en el "Informe Mundial sobre los Salarios 2008/09" ha señalado que durante este año el salario real de miles de personas, en una gran variedad de paises, se reducirá drásticamente, siendo previsible que se produzcan tensiones sociales sobre estos temas.

En este panorama se presenta una cuestión relevante para el Estado: ¿habrá que ampliar la tutela de los trabajadores a expensas de las empresas? ¿Qué ocurriría si se resuelve lo exactamente inverso? De no mediar un manejo adecuado por parte del Estado, el conflicto social 
puede adoptar dimensiones regionales, agravándose la situación en la medida de que los efectos de la crisis se prolonguen en el tiempo. Cobrarían renovados bríos aquellas viejas tesis que propugnaban que la función del Derecho del Trabajo consiste en evitar el conflicto social, una suerte de "Estado de guerra" entre los propietarios y los trabajadores desposeídos.

De acuerdo con Marta Navas-Parejo 4 las típicas respuestas desde el Derecho del Trabajo a las crisis empresariales son concursales o extraconcursales. En lo que refiere a las segundas, habrán medidas que incidan sobre el empleo (regulando el reparto del tiempo de trabajo o aminorando los costos laborales), así como otras que serán propiamente manifestaciones de flexibilidad laboral.

Esta es la clave para comprender a las relaciones laborales de hoy: la flexibilización. En lo que Antonio Baylos 5 ha denominado "culpabilización del Derecho del Trabajo", esta rama del derecho ha sido responsabilizada de los procesos de destrucción del empleo. Hoy en día el Derecho del Trabajo, como conjunto normativo, se legitima en la medida en que procure un nivel aceptable u óptimo de empleo en el país. En ese orden de ideas, la flexibilidad es el nuevo obsesivo fundamento de las relaciones laborales: ella todo lo soluciona.

Como constante presión para reducir la tutela protectora laboral, la flexibilización siempre afectará derechos de los trabajadores: sea en la continuidad de sus contratos o bien en la suma de sus beneficios. Un país puede verse literalmente empujado a dictar legislación que favorezca la flexibilidad o, por el contrario, a proscribirla, según a qué actor social preste atención. Tal vez por ello pareciera que ante los Estados democráticos ningún actor social tiene más poder que otro durante una crisis: las reformas laborales quedan suspendidas.

Si la economía se encuentra en una seria encrucijadà. ¿Habrá que ofrecer más flexibilidad? Desde la perspectiva de las políticas públicas, la unívoca flexibilización es insuficiente para afrontar las dificultades traídas por la crisis, pues cautelaría solo los intereses de un componente del sector privado (el empresariado) dejando de lado a los trabajadores, quienes también lo conforman. ${ }^{6}$

Existen medidas que desde una perspectiva más amplia que el Derecho del Trabajo existen para paliar los efectos en los trabajadores. Teóricamente, algunas de estas medidas pueden ser: la jubilación anticipada y el seguro de desempleo. La primera consiste en adelantar la edad de retiro del trabajador de su vida laboral activa. La segunda, consiste en una prestación sujeta a una temporalidad establecida y el cumplimiento de condiciones que tiene que cumplir quien pretenda ser su beneficiario.

No parecen ser estas soluciones pertinentes (al menos hoy) en la realidad peruana. A continuación, plantearemos aquellas que se presentan con un mayor grado de probabilidad en nuestro medio.

\section{Fomento del diálogo social}

En primer lugar, se debe propiciar que el diálogo social pueda ser la fuente de consensos que afronten las dificultades traídas por la crisis. Con los sindicatos en un reciente y lento resurgimiento, esto es particularmente difícil en una

4 NAVAS-PAREJO, Marta, Medidas de empleo ante situaciones de crisis empresarial. En: Las relaciones laborales en la reestructuración y el saneamiento de empresas, XVI Congreso Nacional de Derecho del Trabajo y de la Seguridad Social, Madrid, Ministerio de Trabajo y Asuntos Sociales, 2006, p. 618.

5 BAYLOS GRAU, Antonio, Creación de Empleo, Mercado de Trabajo y Derecho del Trabajo: el caso español. En: Derecho \& Sociedad, No.30, Asociación Civil Derecho y Sociedad, Lima, Julio de 2008, p. 29.

6 Esto ha sido reconocido recientemente en la VI Reunión Conjunta de los Consejos Consultivos Empresarial y Laboral Andinos - "Declaración de Salinas", del 22 de enero de 2009. 
coyuntura económica negativa, sin que eso pueda significar el cierre de los canales de diálogo.

Aunque en general las experiencias no han sido del todo alentadoras en América Latina (en verdad en Perú ha sido frustrante), es necesario que los gobiernos propicien espacios donde los actores sociales mantengan sus actuaciones en el orden de la concertación, evitando así que el conflicto social, azuzado por la crisis, envuelva a la sociedad por completo.

Desde luego, sería un contrasentido dirigir el diálogo social hacia la formulación de reformas legislativas dado que el consenso en ese caso sería nulo, y si lo hubiera estaría viciado por ambigüedades, referido a aspectos no centrales. Los temas tocados en el diálogo social deberán ser amplios, desde un enfoque interdisciplinario que parta de la necesidad de promover el empleo, al mismo tiempo que salvaguardar la competitividad y productividad de las empresas.

\section{Reducción del Tiempo de Trabajo}

A menos horas de trabajo por trabajador, habrá más trabajo disponible. Con esa fracción de trabajo (horas dejadas de trabajar), se crean nuevos puestos de trabajo, empleando así a más trabajadores. En términos cualitativos, la premisa puede encontrar el éxito en la lucha contra el desempleo. La experiencia francesa en la reducción de la jornada laboral a 35 horas, sin embargo, nos enseña que los resultados pueden ser discutibles.

En favor la reducción de la jornada laboral puede decirse que su aplicación beneficia a aquella fuerza de trabajo que pueda haber perdido su empleo por efectos de la crisis. Por cierto, una medida así debería alcanzar también a aquellos que se encuentran en desempleo permanente.
Pero el presupuesto para el éxito de esta medida es que se mantenga el nivel de producción previo al que existía antes de la reducción efectiva de la jornada. Asimismo, habría que añadir que debe existir una limitación (o eventual supresión temporal) del trabajo en horas extras, como es natural.

En cuanto a los resultados que se puedan obtener mediante la reducción del tiempo de trabajo, pueden ser de lo más variados. Podrían llegar a tener algún efecto paliativo en el contexto de la crisis, pero nos parece que no podría ser aplicada como medida permanente en Perú pues "un desempleo de tipo estructural no es susceptible de reducción significativa por un simple cambio de las horas de trabajo".

\section{Incentivos para no despedir}

Como explica Mario Pasco ${ }^{8}$, hacer más caros los despidos constituye una medida precautoria del empleo, ya que el empleador deberá sopesar los costos involucrados y decidir, desde una óptica estrictamente economicista, si le es más ventajoso prescindir del trabajador, asumiendo el costo del despido, o conservarlo. Entonces la indemnización por despido es una medida legal que obliga al empleador a una ponderación de la relación costo/beneficio.

Este esquema se ve relativizado durante los tiempos de crisis, donde las decisiones económicas suelen tomarse sobre la base de especulaciones e indicadores económicos muchas veces tergiversados. Si la crisis adoptase dimensiones de cuidado, la indemnización por despido puede fácilmente pasar a ser una suerte de "mal menor" que cualquier empresario estaría dispuesto a asumir.

Con la virtual desaparición de la función precautoria de la indemnización por despido,

7 CÓRDOVA, Efrén, La reducción del tiempo de trabajo frente a sus últimas fronteras. En: Revista Trabajo y Seguridad Social, No. 7, El Derecho, Buenos Aires, julio de 2005, p. 586.

8 PASCO COSMÓPOLIS, Mario, Promoción del empleo y protección contra el desempleo. Tesis para obtener el grado de Magister en Humanidades por la PUCP, sustentada en 2007, p. 166. 
medidas imaginativas que partan desde nuevos enfoques pueden tener origen en la sinergia entre el sector privado y el Estado. En algún caso pueden existir subsidios que desincentiven a las empresas de recurrir a ceses colectivos e incluso despidos individuales. Desde luego, este subsidio no necesariamente tomará tal nombre y forma, pues podría presentarse como un beneficio tributario que en la realidad tenga los mismos objetivos.

Cabe apuntar que la terminación del contrato de trabajo por causas objetivas está prevista en el artículo 46 del TUO de la Ley de Productividad y Competitividad Laboral (LPCL), donde se establece como condición el que la medida afecte a no menos del diez por ciento del total del personal de la empresa.

El planteamiento de la ley es discutible, como cada vez que se adoptan porcentajes arbitrariamente: ante un supuesto de fuerza mayor (la crisis) se estaría obligando a los empleadores a reducir personal bajo el amparo de esta causal siempre por encima del porcentaje establecido por ley. Ni un solo trabajador menos. Es previsible que la reforma en este punto sea impulsada por los gremios empresariales. Sin embargo, vista la norma desde el otro lado, la ley asegura a los trabajadores una protección frente a los despidos "sin costo" durante la crisis, que se traduce en un mandato al empleador de aplicar la causal cuando sea realmente necesaria una disminución del número total de trabajadores. El porcentaje de trabajadores que se impone cesar sería un pretexto para evitar que los despidos injustificados, arbitrarios, nulos y fraudulentos se conviertan en justificados con excusa en la crisis

\section{Suspensión perfecta de contratos de trabajo como alternativa al cese del vinculo}

La suspensión del contrato de trabajo "es una de sus contingencias naturales, la más frecuente y necesaria y acaso hasta deseable". ${ }^{\prime 9}$ Un contexto de crisis económica podría bien configurar aquel supuesto.

La LPCL establece en su artículo 11 que es procedente la suspensión perfecta cuando cesan las obligaciones de empleador y trabajador, sin que concluya el vínculo laboral. En el artículo siguiente se establece como causal relevante a nuestro análisis la fuerza mayor y cualquier otro supuestos desarrollado por norma expresa. Creemos que una crisis económica que afecte al mercado se tipifica perfectamente en la primera causal, pero, a efectos de mantener la seguridad jurídica en el país, sería preferible que una existiese una medida legal que lo contemple (segundo supuesto del artículo 12).

Como medida menos gravosa que el despido, podría resultar beneficioso para trabajadores y empleadores que se expidiese legislación de emergencia que regule supuestos en los que, en determinadas actividades económicas, sea posible suspender los contratos de trabajo en vez de recurrir a los despidos. Ni empleador ni trabajador ejecutarian sus prestaciones propias, durante el tiempo en el que se haya previsto para la actividad específica.

El fundamento de esta medida podría sustentarse en la buena fe que debe existir entre las partes en la ejecución del contrato de trabajo. Su eficiencia, sin embargo, está condicionada al desarrollo de la crisis. Si esta afecta a nuestro mercado durante unos pocos meses, esto sería viable. En una eventual profundización de la crisis, los contratos suspendidos ya no tendrían sentido, pues sería ilógico forzar a las empresas a readmitir a los trabajadores cuando ellas están intentando recuperar sus niveles de producción.

Impulso a la constitución de microempresas y de trabajos autónomos

9 PASCO COSMÓPOLIS, Mario, Suspensión del contrato de trabajo. En: Instituciones de Derecho del Trabajo y de la Seguridad Social, Universidad Nacional Autónoma de México, México, 1997, p. 481. 
Los programas del Ministerio del Trabajo y Promoción del Empleo (MTPE) podrían cobrar protagonismo en este sentido. El impulso de formas autónomas de trabajo y emprendimientos puede ser fundamental para vadear los tiempos de crisis.

En la crisis, este planteamiento encuentra serias dificultades. El que una microempresa, por ejemplo, supere la "mortandad infantil" (cerrar sus puertas durante sus primeros años de vida) depende, en buena medida, de que pueda acceder al crédito financiero. Como se ve, esto estaría altamente condicionado por cómo se desarrolle la crisis. En los peores escenarios el crédito se congelaría, condenando a las microempresas y emprendimientos autónomos a una pronta extinción.

Desde luego, la creación de nuevos emprendimientos no necesariamente tendría que ser estimulada únicamente por el Estado. Es posible que también las grandes empresas colaboren en la orientación de sus trabajadores cesados para que constituyan nuevas empresas, pero ello deberá ser supervisado con especial atención por el MTPE pues existe el riesgo (no exclusivo de los tiempos de la crisis) de que ello sea utilizado por las grandes empresas para atomizarse fraudulentamente, evadiendo así a la legislación laboral y la de seguridad social.

\section{La Compensación por Tiempo de Servicios}

Este beneficio laboral, propio del régimen de la actividad privada, es en esencia un mecanismo de ahorro a largo plazo, y un seguro propiamente dicho (distinto al de desempleo), en el que, "mediante el pago de una prima, los individuos tratan de protegerse frente a las consecuen- cias de ciertos eventos, cuyo esperado es no deseado." 10 Implica la formación de un fondo acumulativo en cuentas individuales mediante retenciones mensuales efectuadas a la remuneración del trabajador, el cual puede ser cobrado por él al cese del vínculo.

En los tiempos de la crisis la CTS debería servir para aliviar (en alguna medida) las necesidades de los trabajadores cesados, hasta que pueda reinsertarse en el mercado laboral. Por eso, cualquier propuesta legislativa que intente convertir a la CTS en una suerte de fondo disponible por casi cualquier motivo debe de ser rechazada.

\section{El caso de los Programas de Empleo Temporal}

Mención aparte merece este tipo de programas, instrumento que ya viene siendo aplicado en nuestro país por el MTPE. En abstracto, estos programas buscan satisfacer la demanda de empleo en zonas particularmente afectadas por la escasez de empleo, generando oportunidades laborales en trabajos comunitarios, vinculados al bienestar de la región específica.

El empleo temporal, por definición, es aquel que ocupa a una persona durante 3 o 4 meses. En sí mismo, un programa de estas características no constituye una solución a la escasez de empleo en la crisis, no solo por su carácter temporal, sino por límites inherentes. De acuerdo con Jurgen Weller" el gran problema que estos programas traen consigo es el denominado "encierro del programa", por el cual los participantes que obtienen empleos temporales no logran insertarse al mercado por la ausencia de acertados mecanismos de salida del programa.

10 CHACALTANA, Juan y LÓPEZ, Kristian, ¿Cuánto cuesta un seguro de desempleo? En: Revista Análisis Laboral, No. 139, abril, Lima, 2003, p.11.

11 WELLER, Jurgen, Programas de Empleo Temporal. Algunos aspectos de diseño. Conferencia dictada en el marco del "Seminario Internacional: los Programas de Empleo Temporal y la Crisis Financiera Internacional", celebrado en la ciudad de Lima, Perú, el 9 de diciembre de 2008. 
A decir de Juan Chacaltana ${ }^{12}$, los programas de empleo temporal son mecanismiss anticíclicos. Necesitan ampliarse en período; y lugares en crisis, pero deben de reducirse cuando se experimente crecimiento económico. Este aspecto estructural se complementa con un diseño que atienda a las especificidades del mercado de trabajo (rural o urbano).

Las principales ventajas de los programas de empleo temporal son: costos de' implementación relativamente bajos; un notable impacto múltiple y la contribución que hai-en a las políticas anticíclicas al proteger a grupos vulnerables y estabilizar demandas sociales.

A contrapartida, los problemas relacionados a estos programas suelen ser: una baja remuneración (en Perú está casi por el orden de los 300 soles); el clientelismo; los altos costos que para el aparato estatal suponen el seguimiento del programa; y según lo ha denunciado el sector privado, pueden darse casos de competencia desleal.

Es importante advertir que en ningún caso, los programas de empleo temporal pueden ser paliativos para los despidos colectivos de trabajadores. De ser ello así, se haría más sencillo despedir a los trabajadores.

En lo que refiere a la compensacicin por el trabajo prestado, Juan Chacaltana ${ }^{13}$ ha señalado que debe ser mesurada. No muy baja, como para aliviar sus demandas, ni tan alta, para evitar el efecto de "encierro". Además, el nıonto de compensación debe de ser variable, cle acuerdo a la situación del mercado laboral en cada región del país.

Como decíamos al comienzo, en Perú ya existe el programa Construyendo Perú, que está a cargo del Ministerio de Trabajo y Promoción del Empleo. Su antecedente, denominado "A trabajar urbano" (2001-2006) nació para dar soporte a las familias afectadas por la recesión económica que afectó al Perú entre 1998 y el 2001, ya que no existía un mecanismo de protección social contra el desempleo.

Con el paso de los años, el programa Construyendo Perú ha presentado algunas deficiencias que lo alejan de lo que un Programa de Empleo Temporal debe ser. Para empezar, recién empezó a funcionar en 2002, cuando la crisis a la que pretendía combatir ya había desaparecido. Esto contradice a la teoría, pues estos programas deben de retroceder cuando se experimente crecimiento económico (como ha sucedido en las economías de varias regiones del país desde el año en que empezó sus operaciones). Podemos decir que el Programa ha demostrado una vocación por supervivir que contradice a su instrumentalidad.

Por otro lado, durante su vida el Programa ha venido realizando capacitaciones que se orientan al desarrollo técnico y productivo de los pobladores que participan en sus campañas, pero sin que existan verdaderos planes de reinserción laboral. Existe la necesidad de que el cambio de enfoque en Construyendo Perú parta de reconocer que su auténtico objeto es la creación de empleo temporal, pero con un plan adecuado para la reinserción en el trabajo de sus participantes. El Programa se reduciría a un mero paliativo de la pobreza si sus "empleados-temporales" no tuvieran éxito en encontrar empleo cuando el Programa retroceda, una vez que se cumplan las metas.

Cabe señalar que los mecanismos de salida del programa deben dirigirse no solamente a la población que participa, sino también a la salida del programa mismo. Yes que pareciera que el riesgo de encierro en el programa puede alcanzar también a sus funcionarios. Hay una marcada tendencia a la permanencia del programa, imputable, es cierto, a la amplitud de las demandas sociales, pero también a la burocracia que pretende permanecer en ese puesto de trabajo.

12 Chacaltana, Juan, La evaluación del inpacto del Programa Construyendo Perú. Algunas reflexiones. Conferencia dictada en el marco del "Seminario Intern xcional: los Programas de Empleo Temporal y la Crisis Financiera Internacional", celebrado en la ciudad de Lima, Perí, el 9 de diciembre de 2008.

13 CHACALTANA, Juan, Op. Cit. 
Como reflexión, para responder mejor a la crisis, el programa debería adecuarse al modelo de los Programa de Empleo Temporal. Es importante que exista una reingeniería en la aplicación de Construyendo Perú, para hacer frente a la crisis mediante la generación de productividad en cada región. Entre otras cosas, de la eficiencia de este programa depende que varias regiones del país sorteen con éxito los efectos de la crisis económica.

\section{PERSPECTIVAS PARA EL FUTURO}

El gran desafío al que se enfrenta el Estado consiste en evitar los efectos de la crisis, para lo cual se está sirviendo del crecimiento precedente. En lo laboral, la tarea estatal pasa por no permitir la caída estrepitosa de los niveles de empleo, evitando así que se agraven los conflictos sociales.
Como hemos repasado, existen diversas medidas que pueden ser adoptadas para enfrentar las adversidades que puede traernos la crisis. Desde el Derecho del Trabajo existen respuestas (no todas novedosas) que en común se pueden reducir a un principio de menor afectación al trabajo existente. Flexibilidad y promoción del empleo son premisas a las que el Derecho del Trabajo debe ajustarse, siempre preservando los derechos fundamentales de los trabajadores.

En este contexto, se deberá tener en cuenta que tanto los empleadores como sus trabajadores conforman aquel "sector privado" que merece atención del Estado en la crisis, pero del que casi siempre se habla en abstracto y solo en referencia a las empresas. Mientras tanto los actores laborales deberán fijar sus esfuerzos en recuperarse a prontitud, restableciendo la competitividad del país en el mercado internacional, con lo que se fortalecerá a la economía nacional y con ellas al trabajo. 